\title{
Introductory review
}

\section{The Cosmic Distance Scale: \\ Past, Present, and Future}




\title{
The cosmic distance scale and $\mathrm{H}_{0}$ : Past, present, and future
}

\author{
Wendy L. Freedman \\ The Observatories, Carnegie Institution for Science, 813 Santa Barbara Street, Pasadena, \\ CA 91101, USA \\ email: wendy@obs.carnegiescience.edu
}

\begin{abstract}
Twenty years ago, there was disagreement at a level of a factor of two as regards the value of the expansion rate of the Universe. Ten years ago, a value that was good to $10 \%$ was established using the Hubble Space Telescope (HST), completing one of the primary missions that NASA designed and built the HST to undertake. Today, after confronting most of the systematic uncertainties listed at the end of the Key Project, we are looking at a value of the Hubble constant that is plausibly known to within $3 \%$. In the near future, an independently determined value of $\mathrm{H}_{0}$ good to $1 \%$ is desirable to constrain the extraction of other cosmological parameters from the power spectrum of the cosmic microwave background in defining a concordance model of cosmology. We review recent progress and assess the future prospects for those tighter constraints on the Hubble constant, which were unimaginable just a decade ago.
\end{abstract}

Keywords. cosmological parameters, distance scale, galaxies: distances and redshifts, infrared: stars, Cepheids

\section{Introduction and Motivation}

Tremendous progress has been made over the past two decades in strengthening the foundations of the cosmic distance scale, and in applying both classical and new techniques to the problem of determining distances to galaxies over cosmologically significant scales (for recent reviews, see Jackson 2007; Freedman \& Madore 2010; and references therein). In the final decade of the last century we were still looking at 'a factor of two' uncertainty in the extragalactic distance scale. Then, at the turn of the century, with the publication of the final results from the Hubble Space Telescope Key Project on the extragalactic distance scale (Freedman et al. 2001), that uncertainty dropped to $10 \%$. And now, a decade into the $21^{\text {st }}$ Century, we have good reason to believe that the Hubble constant is known to $3-5 \%$, both in its statistical and systematic uncertainties (Komatsu et al. 2011; Riess et al. 2011; Freedman et al. 2012; Mehta et al. 2012).

The Hubble constant is just one of many parameters describing the basic make-up of the Universe. From analysis of anisotropies in the cosmic microwave background (CMB) and studies of supernovae, baryon acoustic oscillations (BAO), and a number of other experiments, a concordance model has emerged over the past decade. The following set of cosmological parameters is representative of this concordance model (Komatsu et al. 2011; Riess et al. 2011; Freedman et al. 2012): $\mathrm{H}_{0}=73 \pm 3 \mathrm{~km} \mathrm{~s}^{-1} \mathrm{Mpc}^{-1}$, $\Omega_{\mathrm{CDM}}=0.228 \pm 0.027, \Omega_{\Lambda}=0.727 \pm 0.03,-0.0179<\Omega_{k}<0.0081$, and $w=-1.10 \pm 0.14$, where $\mathrm{H}_{0}$ is the Hubble constant, $\Omega_{\mathrm{CDM}}$ and $\Omega_{\Lambda}$ are the mass densities of the Universe contributed by cold dark matter $(\mathrm{CDM})$ and dark energy (DE), respectively, $\Omega_{k}$ is the curvature of the Universe, and $w$ is the equation-of-state parameter.

However, the power spectrum of the CMB does not reveal these parameters unambiguously; their extraction is model-dependent and features across the power spectrum contain varying amounts of information about all of the parameters to be extracted. 
This crosstalk results in cross-correlations and degeneracies among the derived parameters. Most notably, the value of the Hubble constant is strongly degenerate with many of the other derived parameters. Clearly, if the Hubble constant could be independently determined to even higher accuracy, it can act as high-leverage prior on constraining the other terms. An example of this is seen in fig. 19 of Komatsu et al. (2009), where the degeneracy of $\Omega_{\Lambda}$ on $H_{0}$ is shown. This quantitatively underscores the effect of decreasing the uncertainty in an independently determined value of the expansion rate on determining $\Omega_{\mathrm{DE}}$. Increasingly accurate determinations of $\mathrm{H}_{0}$ also constrain the sum of neutrino masses and the numbers of neutrinos, given their contribution to the relative amplitudes of features in the CMB power spectrum shared with $\mathrm{H}_{0}$.

In the following brief discussion, I summarize some recent results from the Spitzer Space Telescope (the 'Carnegie Hubble Project,' CHP), and assess the future prospects for a number of methods for measuring extragalactic distances. A review of recent results from other methods was given in the oral version of this talk; these other topics, however, are covered comprehensively by other authors in this volume and will not be repeated here.

\section{Re-casting the Foundations: Implications of a New Distance to the LMC}

The CHP has been using the Spitzer Space Telescope to recalibrate the Cepheid distance scale in the mid-infrared regime so as to retire the majority of sources of systematic error outlined in the concluding paper of the HST Key Project team (Freedman et al. 2011, 2012). Full $3.6 \mu$ m light curves were obtained for 80 long-period Cepheids in the Large Magellanic Cloud (LMC) and for all 10 of the Galactic Cepheids for which Benedict et al. (2007) obtained direct trigonometric parallaxes using the HST/Fine Guidance Sensor. Extinction corrections in the mid-IR range as compared to the $V$ band are reduced by more than an order of magnitude, and metallicity corrections are empirically found to be small. The resulting observed scatter in the mid-IR period-luminosity relation is only $0.10 \mathrm{mag}$.

These mid-infrared observations give rise to a new determination of the distance modulus to the LMC of $18.477 \pm 0.033 \mathrm{mag}$ (Freedman et al. 2012; Monson et al. 2012). Leavitt relations at $3.6 \mu \mathrm{m}$ for the LMC and Milky Way Cepheids are shown in Fig. 1. When used to recalibrate the Key Project results and also tie in to more recent supernova data at cosmologically significant distances (i.e. minimally impacted by large-scale flows), a value of the Hubble constant of $\mathrm{H}_{0}=74.3 \pm 2.1 \mathrm{~km} \mathrm{~s}^{-1} \mathrm{Mpc}^{-1}$ (systematic uncertainty) is obtained. This systematic error is formally at the $2.8 \%$ uncertainty level (see Fig. 2). As described in Freedman et al. (2012), this value of the Hubble constant, when combined with WMAP7 data, yields a value of the equation-of-state parameter, $w_{0}=-1.09 \pm 0.10$ (see Fig. 3). Relaxing constraints on flatness and combining $\mathrm{H}_{0}$ results with those of WMAP7, Type Ia supernovae (SNe Ia), and BAO yields a value for the effective number of neutrino species of $N_{\text {eff }}=4.13 \pm 0.67$, mildly consistent with the existence of a fourth neutrino species (see Fig. 4). These results are consistent with a number of recent studies including Benson et al. (2011), Keisler et al. (2011), Mehta et al. (2012), and Riess et al. (2011).

Very similar values of $\mathrm{H}_{0}$, ranging from 70 to $74 \mathrm{~km} \mathrm{~s}^{-1} \mathrm{Mpc}^{-1}$, have been published recently, i.e. $\mathrm{H}_{0}=70.2 \pm 1.4 \mathrm{~km} \mathrm{~s}^{-1} \mathrm{Mpc}^{-1}$ from analysis of WMAP7 data (Komatsu et al. 2011), $\mathrm{H}_{0}=70.1 \pm 1.5 \mathrm{~km} \mathrm{~s}^{-1} \mathrm{Mpc}^{-1}$ from BAO (Percival et al. 2010), $\mathrm{H}_{0}=73.8 \pm$ $2.4 \mathrm{~km} \mathrm{~s}^{-1} \mathrm{Mpc}^{-1}$ from SNe Ia (Riess et al. 2011), and $\mathrm{H}_{0}=74 \pm 6 \mathrm{~km} \mathrm{~s}^{-1} \mathrm{Mpc}^{-1}$ based on a calibration of nuclear masers (Macri et al. 2006). All of these values agree to within 


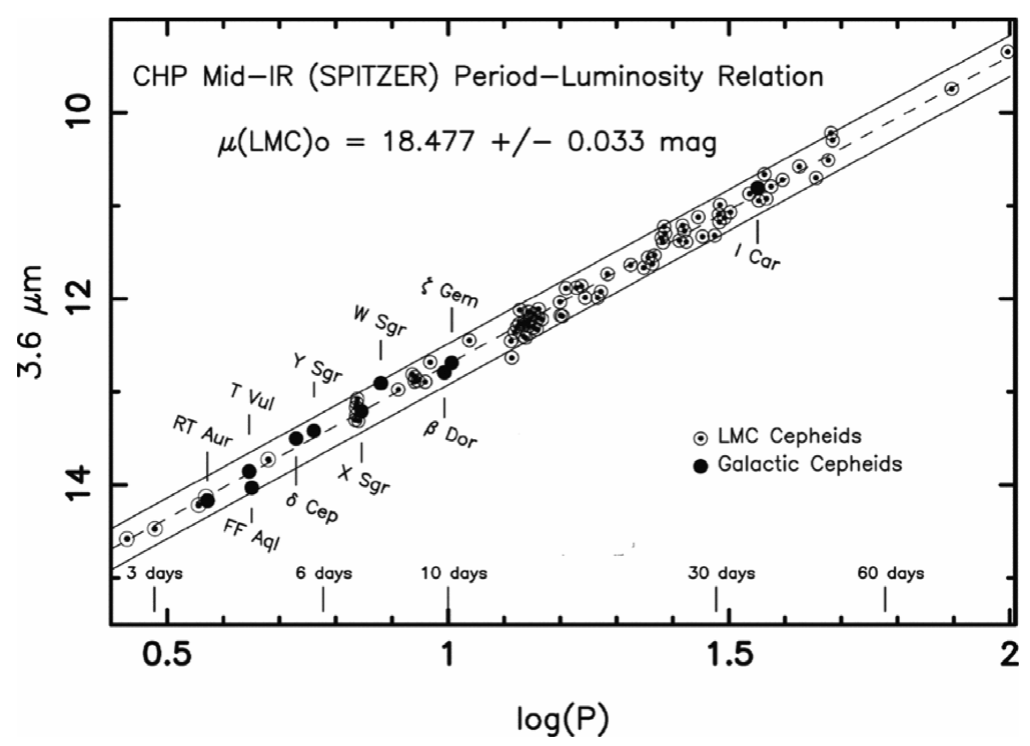

Figure 1. Mid-infrared $3.6 \mu \mathrm{m}$ period-luminosity relation (Leavitt law) for 80 Cepheids in the LMC (Scowcroft et al. 2012) - small circled points-calibrated using 10 Milky Way Cepheids (Monson et al. 2012) - individually marked large filled circles - with HST trigonometric parallaxes. The slope of the Leavitt relation is set by the LMC sample. The dashed slope is defined by the sample of 80 LMC stars; the solid lines are $2 \sigma$ ridge lines. See Freedman et al. (2012) for details.

$1 \sigma$ with the $2001 \mathrm{Key}$ Project value of $72 \mathrm{~km} \mathrm{~s}^{-1} \mathrm{Mpc}^{-1}$. These latest values of the Hubble constant bolster our confidence that convergence is occurring. The new measurements agree to within $0.5 \sigma$ with the 2001 value, as scaled by the Key Project's originally quoted $8 \mathrm{~km} \mathrm{~s}^{-1} \mathrm{Mpc}^{-1}$ uncertainty.

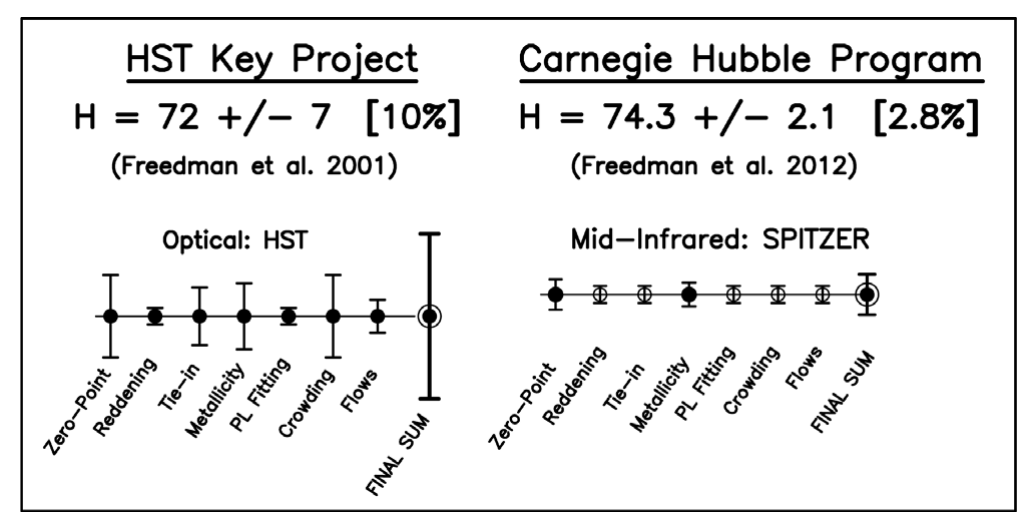

Figure 2. Graphical illustration of the dramatic decrease in each of the seven dominant sources of systematic error in determining the Hubble constant. The left-hand panel graphic the individual errors given for the HST Key Project by Freedman et al. (2001; filled circles), followed by the total systematic error formed from the quadrature sum of the six preceding values (circled dots). The right-hand portion shows the current errors for the same terms for the CHP determination of the Hubble constant. See Freedman et al. (2012) for details. 


\section{Re-casting the Foundations: Astrometry}

Gaia - Nothing is more fundamental to the extragalactic distance scale than having direct trigonometric parallaxes to stars like Cepheids, RR Lyrae variables, and the tip-of-thered-giant-branch (TRGB) population. The European Space Agency's Gaia satellite is currently scheduled for launch in late 2013. It will systematically survey the entire sky with an astrometric accuracy of a few micro-arcseconds and will provide $1 \%$ parallaxes out to several kiloparsecs around the Sun. Hundreds of Cepheids and thousands of RR Lyrae variables will have their parallaxes measured, and a direct distance to the LMC good to approximately $2 \%$ is anticipated (Mignard 2004). It will take the better part of this second decade to get the final results from Gaia, but the results are eagerly anticipated.

$H S T$ - In the mean time, in a series of papers, Benedict and collaborators have used the HST/Fine Guidance Sensor to determine parallaxes to 10 of the nearest Cepheids (Benedict et al. 2007) and four of the nearest RR Lyrae variables (Benedict et al. 2011). These have allowed for the re-determination of the zero points for the Population I and II period-luminosity relations for Cepheids and RR Lyrae stars, respectively.

\section{The Future}

The Dark Energy Task Force (Albrecht et al. 2006) developed a Figure of Merit (FOM) for a number of proposed future stages in determining cosmological parameters. The continuing importance and impact of the Hubble constant on these efforts is summed up by the following quote from Weinberg et al. (2012): "In all cases, adding a prior from an independent measurement of $\mathrm{H}_{0}$ with $1 \%$ accuracy increases the FOM by $40 \%$."

In February 2012, a three-day workshop was held at Stanford University to address the feasibility of attaining a 1\% determination of the Hubble constant. The results were published in the form of a White Paper (Suyu et al. 2012) with the general concensus being that of the methods currently being considered (i.e. those that individually had demonstrable internal errors of less than 10\%), there were several methods identified

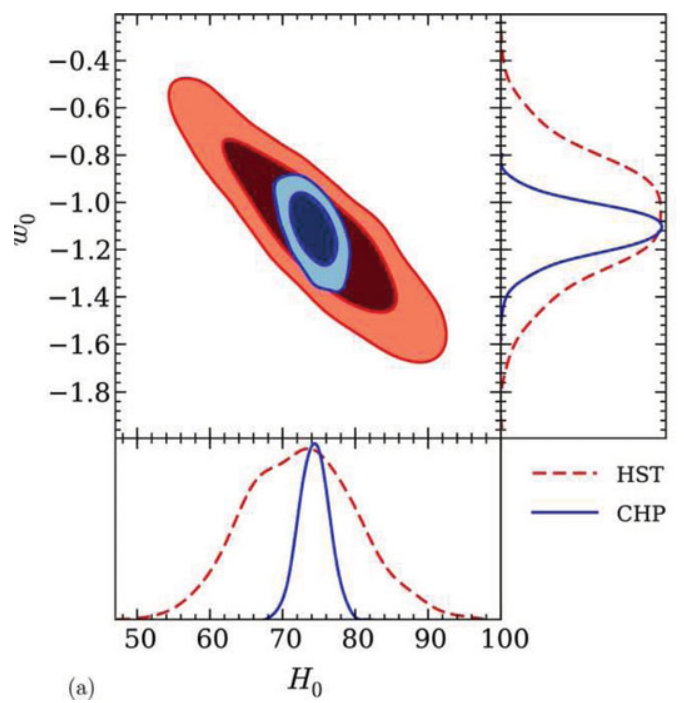

Figure 3. Confidence plots of the equation-of-state parameter, $w_{0}$, constrained by the Hubble constant, $\mathrm{H}_{0}$, using WMAP7 data, assuming $\Omega_{k}=0, w_{a}=0$, and $N_{\text {eff }}=3.046$ (see Freedman et al. 2012 for details). 
that had the potential to deliver an uncertainty in $\mathrm{H}_{0}$ on the order of $1 \%$. A number of methods are briefly discussed below, and a brief assessment of their potential accuracies is given.

- The TRGB method applied to individual galaxies appeared to have a $5 \%$ intrinsic uncertainty, and although it is limited to nearby galaxies, it is clearly a valuable independent check on the Cepheid distance scale.

- The Cepheid period-luminosity relation, in the infrared, has a dispersion of $0.1 \mathrm{mag}$ (or 5\% in distance; Scowcroft et al. 2011), making 1\% distances to individual galaxies and a calibration of the Hubble constant to that same accuracy achievable in principle, assuming that systematic errors can be brought down to this level.

- $\underline{\mathrm{SNe} \text { Ia }}$ can deliver $10 \%$ distances to single objects when observed in the red (Folatelli et al. 2009; Hicken et al. 2009; Riess et al. 2011), and a 1\% value of the Hubble constant is feasible in principle. The biggest challenge is the current dearth of Cepheid zero-point calibrators. Eight SNIa galaxies with Cepheids are currently known; new ones are being found at the rate of only one every $2-3$ years. However, ultimately this sample will increase over time.

- The Tully-Fisher relation and the Fundamental Plane both have a scatter of $20 \%$ for individual galaxies. Averages over very large samples are required to reach $1 \%$ precision, and their reach is not as distant as for supernovae.

- Surface brightness fluctuations (SBFs) distances have random errors of $0.06 \mathrm{mag}$, and a systematic error in the zero point of 0.08 mag (Blakeslee et al. 2010). With larger samples and an improved Cepheid calibration, accuracies of better than a few percent could possibly be achieved. The method is limited to relatively nearby galaxies.

- Planetary nebula luminosity function (PNLF) distances agree extremely well in a relative sense with SBF distances, to 1.5\% (Blakeslee et al. 2010), but the SBF distances

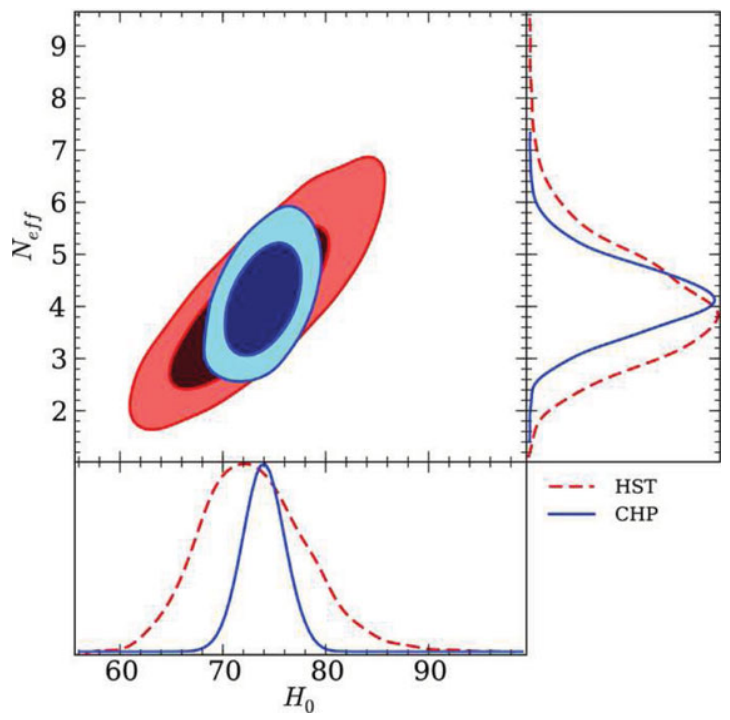

Figure 4. Two-dimensional confidence plot of the effective number of neutrinos, $N_{\text {eff }}$, and the Hubble constant, $\mathrm{H}_{0}$, combining the WMAP7 and BAO data and assuming $\Omega_{k}=0, w_{a}=0$. The red and blue contours show the results using the priors from Freedman et al. (2001) and Freedman et al. (2012; labeled CHP), respectively. The right-hand and bottom panels show the one-dimensional marginalized posterior probability distributions (PPDs) for $N_{\text {eff }}$ and $\mathrm{H}_{0}$, respectively. The Freedman et al. (2001) PPDs are plotted as red dashed lines and the CHP PPDs are plotted as blue solid lines. 
are systematically offset from those of PNLF distances by approximately $15 \%$. The PNLF can provide distances out to some $20 \mathrm{Mpc}$ (Ciardullo 2012).

- Nuclear masers orbiting central black holes in nearby galaxies have geometric distance errors on the order of $10 \%$ (see Lo 2005), but given their scarcity in the currently accessible volume of space, it was not clear how this method could get down to $1 \%$.

- Gravitational-lens time delays are currently giving values of $\mathrm{H}_{0}$ good to $7 \%$ (see Treu 2010; Suyu et al. 2011). Comparison of multiple lines of sight is thought to be essential to understand the systematics.

- CMB studies of the power spectrum, it was felt, yield more information on the cosmological parameters if an accurate value of $\mathrm{H}_{0}$ could be measured independently, rather than the other way around.

- $\underline{\mathrm{BAO}}$ provide a physically well-understood and calibrated 'standard ruler.' A $1 \%$ uncertainty in $\mathrm{H}_{0}$ based on this method appears to be feasible. However, it needs to be more widely emphasized that both $\mathrm{BAO}$ and $\mathrm{CMB}$ 'determinations' of $\mathrm{H}_{0}$ are deeply 'theory-laden,' given that neither method actually measures the Hubble constant at large redshifts but rather they each predict it within the strict context of a given cosmological model (Suyu et al. 2012).

\section{Summary}

Accurate distances are key for probing some of the most fundamental questions about the nature of stars, galaxies, and the Universe. A wide range of new experiments on the ground and missions in space, undertaking fundamental astrometry (Gaia), working at infrared, millimeter, and submillimeter wavelengths (e.g., Planck, JWST, and WFIRST), pursuing DE missions, and employing the next generation of very large optical telescopes (including LSST, E-ELT, GMT, and TMT) all promise to bring the uncertainties in the Hubble constant in particular and the rest of the fundamental cosmological parameters in general, down to unprecedented levels of precision and accuracy, undreamt of even just 10 years ago. There is a realistic possibility that achieving a measurement accuracy of $\pm 1-2 \%$ in the expansion rate of the Universe can be attained in the next decade.

\section{Acknowledgements}

An introduction to this meeting on the Cosmic Distance Scale cannot be complete without acknowledging the passing of two major figures in the field. After spending a lifetime pursuing a value of the Hubble constant, Carnegie astronomer Allan Sandage died in California on 13 November 2010 at the age of 84. This was preceded by the death of our Key Project colleague and long-time friend, Harvard astronomer, John Huchra, who, at the age of 61, died at his home in Cambridge, Massachusets, on 8 October 2010. Both men lived to see the convergence; both men left an indelible mark on it. This would not have been the same journey without them. I gratefully acknowledge my many distance-scale collaborators over the years; those of the Key Project team and the CHP. Most particularly, I thank Barry Madore for his invaluable contributions.

\section{References}

Albrecht, A., Bernstein, G., Cahn, R., et al. 2006, Report of the Dark Energy Task Force, astro$\mathrm{ph} / 0609591$

Benedict, F., McArthur, B. E., Feast, M. W., et al. 2007, AJ, 133, 1810

Benedict, F., McArthur, B. E., Feast, M. W., et al. 2011, AJ, 142, 187

Benson, B. A., de Haan, T., Dudley, J. P., et al. 2011, ApJ, submitted (arXiv:1112.5435) 
Blakeselee, J. P., Cantiello, M., Mei, S., et al. 2010, ApJ, 724, 657

Ciardullo, R. 2012, Ap\&SS, 341, 151

Folatelli, G., Phillips, M. M., Burns, C. R., et al. 2009, AJ, 139, 120

Freedman, W. L., Madore, B. F., Gibson, B. K., et al. 2001, ApJ, 553, 47

Freedman, W. L., Madore, B. F., Scowcroft, V., et al. 2011, AJ, 142, 192

Freedman, W. L., Madore, B. F., Scowcroft, V., et al. 2012, ApJ, 758, 24

Freedman, W. L. \& Madore, B. F. 2010, ARA $\& A$, 48, 673

Jackson, N. 2007, Liv. Rev. Rel., 10, 4

Hicken, M., Wood-Vasey, W. M., Blondin, S., Challis, P., Jha, S., Kelly, P. L., Rest, A., \& Kirshner, R. P. 2009, ApJ, 700, 1097

Keisler, R., Reichardt, C. L., Aird, K. A., et al. 2011, ApJ, 743, 28

Komatsu, E., Smith, K. M., Dunkley, J., et al. 2011, ApJS, 192, 18

Lo, F. 2005, ARA\&A, 43, 625

Macri, L. M., Stanek, K. Z., Bersier, D., Greenhill, L. J., \& Reid, M. J. 2006, ApJ, 652, 1133

Mehta, K.T. 2012, MNRAS, submitted (arXiv:1202.0092)

Mignard, F. M. 2004, Bull. Am. Astron. Soc., 36, 858

Monson, A., Freedman, W. L., Madore, B. F., et al. 2012, ApJ, 759, 146

Percival, W. J., Reid, B. A., Eisenstein, D. J., et al. 2010, MNRAS, 401, 2148

Riess, A. G., Macri, L., Casertano, S., et al. 2011, ApJ, 732, 129

Scowcroft, V., Freedman, W. L., Madore, B. F., et al. 2011, ApJ, 743, 76

Treu, T. 2010, ARA\& $A, 48,87$

Suyu, S. H., Hensel, S. W., McKean, J. P., et al. 2011, ApJ, 750, 10

Suyu, S. H., Treu, T., Blandford, R. D., et al. 2012, arXiv:1202.4459

Weinberg, D. H., Mortonson, M. J., Eisenstein, D. J., et al. 2012, Phys. Rep., in press (arXiv:1201.2434) 